Shakir A. Mehdiyev

DOI: 10.25045/jpit.v08.i1.10

Institute of Information Technology of ANAS, Baku, Azerbaijan

shakir@iit.ab.az

\title{
COMPUTER SYSTEM'S MAINTENANCE IN A CORPORATE ENVIRONMENT
}

The article analyzes the various aspects of the maintenance strategies and their applicability to computer systems. It seeks to review the stages of diagnosis and technical maintenance indicators. The types of maintenance activities are put forth in the computer systems.

Keywords: maintenance, corporate information system, computer system, failures, malfunction, diagnosing, CRM, social engineering.

\section{Introduction}

Created in the middle of the XX century, computers and computer technology undergone a rapid rise in its development, and became an integral part of our daily lives. Complex systems with the use of computer technologies cover many activities in manufacturing, finance, science and medicine. In such systems, the units (enterprises), which are homogeneous in composition and activities, unify in corporate information system (CIS) aiming at the effective functioning.

CIS is considered to be an information system, which includes a set of hardware, communication network, database, document management subsystem, etc. [1].

The most important resource of CIS is the information, the quality of which resolves the implementation of tasks to achieve the final result. The quality of information means the combined implementation of its following properties, namely, completeness, reliability, timeliness, safety and storage reliability. Achieving the goals requires the functioning of CIS, when the requirements of maximum reliability are provided, since the system errors turn into losses of various kinds (sometimes irreparable). In this case, it is necessary to ensure the reliability of individual properties such as reliability, maintainability, storage ability, durability, which are realized through the maintenance and repair (MR).

\section{MR systems}

With regard to the Azerbaijan National Academy of Sciences (ANAS), its CIS structure is a complex system that combines networking, computing and information resources of research institutions of ANAS. The objectives of such a system include a joint effective activity in the virtual medium of research organizations, groups and scientists, as well as improving the efficiency of scientific management and research, the development of all areas of science meeting the modern world standards and the integration into the world scientific environment [2].

CIS of ANAS has been built based on scientific computer network AzScienceNet, which is currently combining more than 4,200 computers, which operate in the research institutes and structures of ANAS [3].

The demand for MR in this environment is evidenced by the results of monitoring conducted in 2013-2014 to ensure computers in ANAS (Fig. 1). The comparative analysis of these results shows a significant increase in the park of computer systems (CS) and, consecutively, an increase in jobs and the tightening of the requirements for MR. 


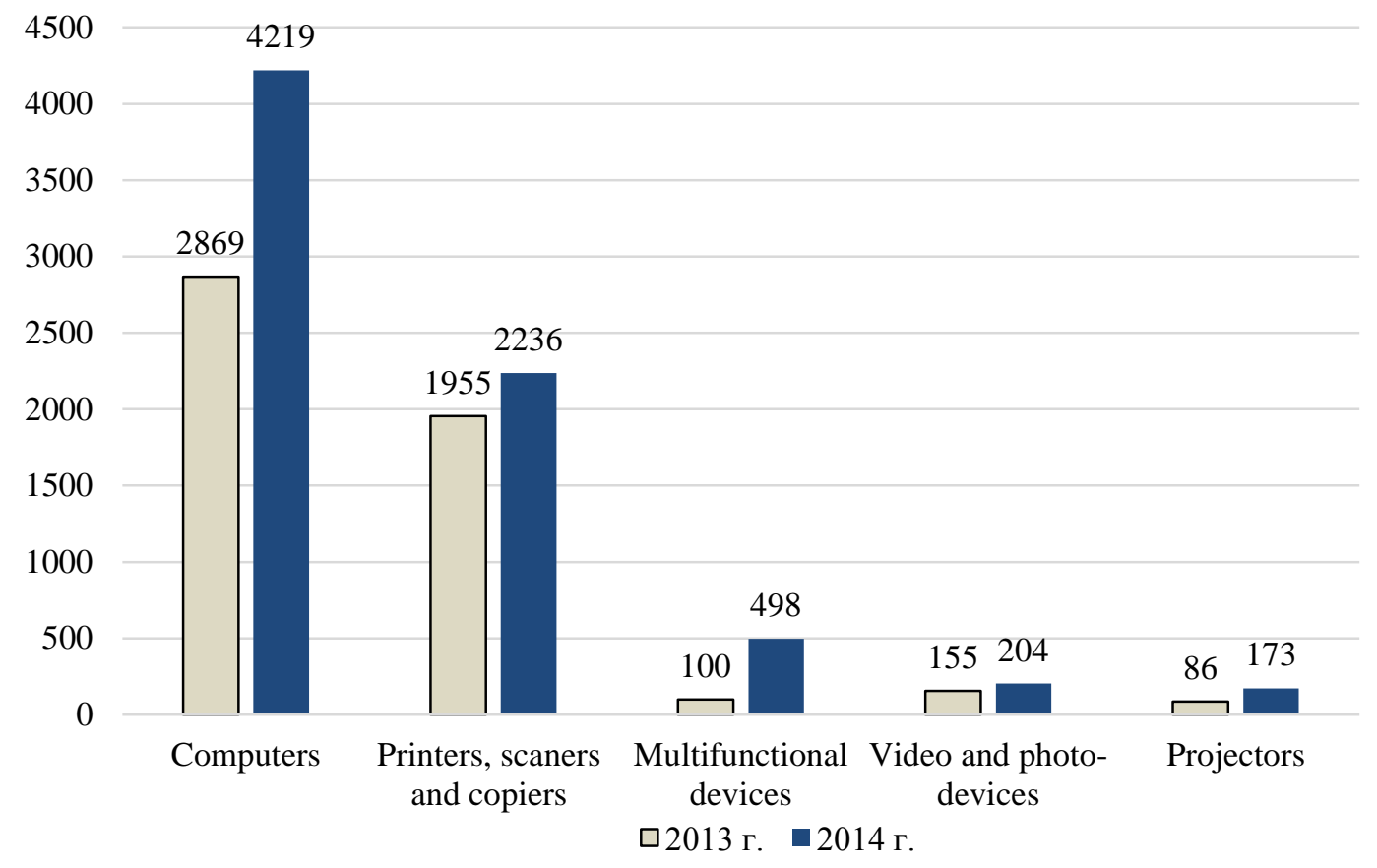

Fig. 1. The provision of computers and peripheral devices in ANAS

The core of AzScienceNet is the data center, which includes a set of engineering infrastructure, software and hardware data processing servers, storage devices, communication links and equipment to provide multiple services to users (hosting, data storage, cloud service, email, e-library, roaming-service of the academic networks, etc.).

The operation of such system meets the standards of reliability, availability and serviceability, which were set up in it during the design and utilization.

However, as a result of utilization, the system is exposed to both deterministic and random influences that lead to the disruption of the system performance. In order to maintain the system in a working condition, to reduce the failure rate and warn about the malfunctions, the system maintenance is carried out in accordance to the standards of some countries [4-6], focus on:

- Identifying and evaluating the true state of hardware system;

- Taking measures to preserve the working condition of the system hardware or to restore them.

Actually, the service system consists of service provision and technical diagnostics of equipment.

Service provision implies the timely supply of consumable materials, replacement of resources, performance of maintenance, and repair work to ensure uninterrupted and high quality performance of CS, which is included into CIS [7].

The main purpose of technical diagnostics is an organization of the effective check of the functioning and operability of CIS and its constituent modules and nodes. Based on the obtained diagnostic information, the decisions are made to ensure the functioning of the CIS (transmission processes, data processing and storage).

There are basic known strategies of MR systems [8-10]:

- Maintenance in case of failure known as Reactive Maintenance. It is used whenever the object deterioration causes a functional failure.

The strategy is based on the principle of fast troubleshooting without examining the root causes of their occurrence. It is used if the repair cost is relatively low.

With regard to CS the failure, which leads to malfunction, may occur, for example, because of the high temperature and humidity, over-current and voltage, vibration, and mechanical impacts. 
At the same time, the failure of the CS and its separate units is often caused not only due to physical malfunction, but also their incompatibility or incorrect software configuration, use of algorithms that are sensitive to violations of information processes.

There are error facts in the design of microprocessors omitted by the developers that do not affect the operation of the standard software and have been discovered by chance in the course of complex calculations [11].

- Preventive maintenance or service under the rules is based on the assumption that there is a causal link between routine maintenance and operational reliability. It is assumed that the statistics on the failure rate $\lambda(t)$,which is accumulated as a result of operating, allows you to accurately calculate the reliability and to determine the time interval, during which the equipment should be repaired:

$$
\lambda(t)=\frac{n(t)}{[N-n(t)] \Delta t}
$$

Where $n(t)$-is the number of failures during the time interval $\Delta t ; N$ - number of reviewed components or products.

At the same time, many elements or nodes of CS become outdated before their physical wear and pulled out of service without a set of sufficient statistical data on their reliability.

The traditional probability model of occurrence of failures is shown in Fig.2. This model is more valid for simple types of equipment.

The components of modern CS implemented based on the theory of physics of semiconductors, have a low failure rate [12]. Reliable data on their reliability can be obtained after long trial periods (hundreds of thousands or even millions of hours of work).

For example, a media player of Spinetix series, which exclusively consists of electronic components, is provided with a lifetime warranty.

But the failures of the components may also be associated with the process of their manufacture, when the running operations - electrical thermal, Heat Soak, thermal cycling, vibration are not applied to reduce the cost of production in the fabrication process [13].

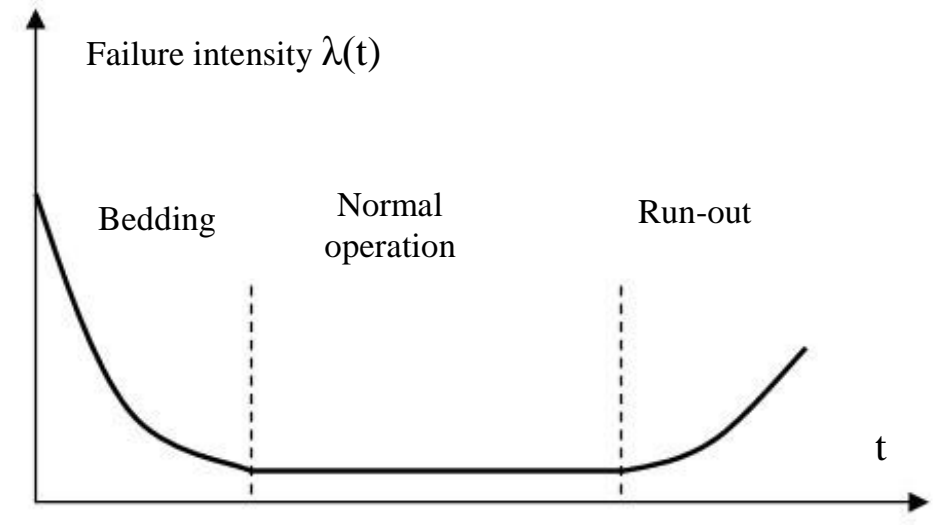

Fig. 2. The model of failure occurrence

As for the software component of CS, it is not subject to physical, and its reliability is not a function of time. When introducing the new generation hardware the previous versions of software very often become inoperable and require an update or correction. Up to the contrary, new software versions are not supported on older hardware.

The reasons for software failure of nonphysical nature may be unintended (inadvertent, accidental) threats caused by the errors occurred when designing the information system and its elements, in the actions of personnel, and deliberate (intentional) threats realized with illegal purposes.

The failures are also caused by overloading of communication networks.

- Condition Based Maintenance, Condition Monitoring Maintenance or Predictive Maintenance involves monitoring and diagnosis of the real condition of the equipment with the help 
of measuring tools, and further, the predictions about the feasibility of future maintenance or repair work are made basing on the mathematical apparatus, avoiding functional failure of hardware. Depending on the used mathematical apparatus the following basic predictions are distinguished [14]:

- expert evaluation, when the expert's opinion on the future condition of the equipment is collected through a survey or questionnaire, then processed and a forecast is achieved;

- analytical prediction, when the amount of controlled parameters characterizing the condition of the equipment by time is determined as a result of prediction;

- probable prediction, when the probability of the condition parameters of the equipment going (not going) beyond the allowed limit is determined as a result of the prediction;

- statistical classification (pattern recognition), when the class of diagnosed object on performance criteria is determined as a result of the prediction.

Hardware and software monitoring of the condition of separate components of CS is realized through log files and graphics, such as the temperature of CPU, motherboard, drives on the HDD hard disk, power supply; supply voltages; fan speed; HDD working surface scanning for physical malfunctions and logical errors (Self Monitoring And Reporting Technology), etc.

- Preventative maintenance or Proactive Maintenance is implemented as a systematic identification and elimination pre-failure situations (potential failure), which can cause unstable working conditions. For example, a periodic dust removal to improve heat dissipation from the processor; identification of specific sounds preceding the pre-emergency status of cooling fans; deletion of temporary files; using anti-virus software; software update.

- Reliability Centered Maintenance is implemented basing on the evaluations of the consequences of failures and the functional importance of the system components, that is, it is focused on ensuring the reliability of the units or nodes of the equipment, which are critical for the system operation. In this strategy, a failure means any unsatisfactory condition of the system, when a function loss (the system stops working) or acceptable quality loss (the system is running, but ergonometric parameters are worsen) occurs. The methods of Failure Mode and Effects Analysis (FMEA) and Fault Tree Analysis (FTA) are used as a tool set.

If certain failures of units or nodes of CS equipment do not affect the performance of the system, it makes no sense to spend resources to prevent all failures, and it is expedient to restore them after functional failure.

A system service TPS (Toyota Production System) is also known, an important part of which is Total Productive Maintenance (TPM) [15]. It introduces the concept of combining the equipment maintenance process with the production process to eliminate the malfunction and losses in the manufacture of high-quality products. Since the system mainly focuses on the prevention and early detection of equipment defects, then according to this approach, TPM can be attributed to the Condition Centered Maintenance.

Analysis of the major maintenance and repair strategies shows that each of them has features that limit the scope of their effective application depending on the functional essence, purpose and conditions of operation of technical equipment in various industries, particularly, in CIS.

Within CIS, the used CS must be differentiated by the extent and intensity of their use in the organizational processes and the potential damage in case of failure.

The task of choosing the service strategy occurs in the case of limited material resources and depends on the functional purpose of the system. So, in our case, the main criteria for assessing the quality of CIS are the availability time and readiness coefficient that determine the effectiveness of information service delivery to the system users.

\section{Diagnosing CS}

$\mathrm{CS}$ is a combination of hardware and software, which are affected by numerous factors. To predict the development of the problem and to prevent its onset, it is necessary to evaluate these factors and make a diagnosis. The action of maintenance services to ensure fail-safe operation of CS will 
depend on this. Technical personnel needs reliable information about a specific unit of service, on the basis of which the problem is revealed and identified, and a plan is prepared and implemented.

The process of CS diagnosing is not included into the traditional formal schemes and can be performed as follows.

Stage One: The monitoring of the current technical condition of CS. For the collection and systematization of the information the direct dimensions of the characteristics, built-in diagnostic programs, a visual inspection or the information about the relationship with customers for further analysis of results (Customer Relationship Management, CRM) are used.

In most cases, users (clients) are direct witnesses, and sometimes the perpetrators of origin of pre-error conditions or equipment disruptions and can be a source of subjective information for diagnosis. In order to effectively survey of users (clients) social engineering techniques can be used, when the user itself can be a source of the most useful and effective information to solve the diagnosing problem by the maintenance services. Technical documentation and maintenance history can be an important source of information.

Step two: setting a working hypothesis of diagnosis through analyzing the measurement results, additional software testing, findings and failure symptoms. At this stage, the lack of information or knowledge acquired in practice can lead to erroneous establishment of fundamental relationships and generalizations. This is a factor of "illusory correlation", when the technical staff produces decision making skills based on individual experience, which has been used successfully in previous practices [16].

Step three: probabilistic analysis of the problem and ignoring improbable events. Different analysis methods are used to understand how malfunction occurs in a complex system and to identify the way to reduce the risks. Currently, Data Mining is developing, the methods of which (clustering, neural networks, fuzzy logic, and others.) provide knowledgeable decision on the optimal effects at MR in a short period of time.

Step four: identification and localization of hardware or software component faults. At this stage, a set of measures aimed at restoring the operating condition of the system is taken. If the problem is localized to within a subsystem, then the decision is made on its adjustment or replacement by the reserve without disrupting operation of the entire system. Next steps involve localization of faults at the element level or device modules and the feasibility of the repair within a limited time interval. After eliminating the malfunction symptoms, the device goes back to operation.

\section{Maintenance indicators}

Maintenance process can be assessed by quantitative and qualitative indicators.

Quantitative indicators include technical and economic indicators, defined by the total time spent for MR; by the average time and frequency of the performance of one type of service; by the cost and the amount of reserved accessories and consumables consumed in the process of maintenance; by the technical staff load factor.

A set of services is assessed by the generalized indicator of the service rate determined by the formula [17]:

$$
\eta=\frac{\sum_{i=1}^{n} t_{\mathrm{i}}}{\sum_{i=1}^{N} t_{\mathrm{i}}}
$$

where $\eta$ - denotes service rate in $\% ; n$ - the actual number of the provided services; $N$ - the theoretical amount of the provided services; $t_{i}$ - time to perform each service $i$.

The quality of service is an intangible indicator of providing qualified recovery service of the served object and it is a collection of MR features, reflecting the satisfaction rate of a potential customer. It may be compliance with regulatory deadlines; friendly and attentive service; timely informing the customer about the changes made to the work completion timing; the competence of personnel and the rate of the used technology; ensuring confidence-building measures within 
the framework of the preservation of confidential information on data carriers; friendly feedback interface for subsequent proper operation of equipment. To be precise, the improvement of the service and the culture of maintenance can be achieved through personal interaction with the customers. One of the tools of organizing the personal contact with the customer is the call-centers and multimedia contact-centers, where the client may apply for maintenance service in a convenient way - by calling, sending an e-mail or fax, visiting the site and contacting the operator [18]. The abovementioned criteria form the reputation concept, i.e. a specific opinion about the ongoing maintenance.

\section{Maintenance Activities in COP}

Activities within the framework of the MR strategies can be classified as follows:

- Maintenance;

- Ongoing repair;

- Overhaul;

- Modernization;

- Replacement and installation of new equipment.

MR experience shows that the most frequently provided services are:

- installation, configuration and recovery of the operating system;

- optimization of the operating system settings;

- installation/configuration of software;

- elimination of hardware and software conflicts;

- regular maintenance of hard disks;

- backup and recovery of data;

- troubleshooting;

- elimination of anti-virus;

- repair and modernization of computers, laptops, servers, etc .;

- replacement/installation of components;

- computer assistance;

- Internet connection setting;

- communication equipment setting;

- connection of peripheral devices;

- preventive measures.

\section{Conclusion}

The article analyzes the main MR strategies. It is shown that different variations of strategies aimed at improving the functioning of the system is used in case of the MR in CN. Peculiarities of $\mathrm{CN}$ diagnosis and the factors influencing the diagnosis are shown; indicators of maintenance are reviewed. Types of MR activities and services in $\mathrm{CN}$ are proposed.

\section{References}

1. Dodonov A.G., Fleitman D.V. Corporate information systems: ensuring survivability // Mathematical Machines and Systems 2005, No4, pp.119-130.

2. Alguliyev R.M., AlakbarovR.G, Aliguliyev R.M., Fataliyev T.Kh. E-science: state-of-the-art, problems and development prospects. Baku: Institute of Information Technology, 2015, p. 127

3. Alakbarov R.G, Fataliyev T.Kh., Mehdiyev Sh.A. Monitoring of science. Baku: Institute of Information Technology, 2015, p.74

4. BS 8210: 2012 Guide to facilities maintenance management.

5. DIN 31051 Fundamentals of maintenance.

6. GOST 18322-78 Maintenance and equipment repair system. Terms and Definitions. 
7. Volkov V.Y., Veprentseva O.N. Service and diagnostics of computer and microprocessor systems. Novomoskovsk, 2009, p.68

8. Shehvatov D. Evolution of management systems for maintenance and equipment repair// (supplement to the journal "Expert"), 2004, pp.18-24.

9. Antonenko I.N., Kryukov I.E. Information systems and practices MR: development stages// Chief Engeneer 2011, No10, pp.37-44.

10. NASA Reliability Centered Maintenance Guide for Facilities and Collateral Equipment, 2000, $348 \mathrm{p}$.

11. www.hi-news.ru/computers/intel-priznala-nalichie-kriticheskoj-nedorabotki-v-processoraxskylake.html

12. MIL-HDBK-217F. Reliability prediction of electronic equipment, in 1991.

13. Blintsov A.E., Mozhenkova E.V.et al. The use of index of data loss due to failure to assess the rate of data security / Proceedings of the XVI scientific conference, Grodno, 2011, pp.174-176.

14. http://servisv.org.ua/articles/sovremennye-sredstva-i-metodiki-diagnostiki-oborudovanija

15. Ichikawa A., Takagi I. et al. MR in a simple and accessible presentation. M .: RIA "Standards and Quality", 2008, p.128

16. Zlatin P.A., Krekova M.M., SokolyanskyV.V. Sociology and psychology of Labour. Ed. Zlatin P.A. - Part 2 M.: MSIU 2008, p.239

17. Sahno E.Y., Dorosh M.S., Rebyonok A.V. Service Management: Theory and Practice: Training Manual. K.: Center for educational literature, 2010, p.328

18. Bukashin D. How is an effective contact-center? // Technology and communications, 2013, No6, pp.38-39. 